\title{
Waiting for 4.1
}

\section{Review of: Roche Genetics: Education Program, CD-ROM version 4.0; F. Hoffmann-La Roche; www.rochegenetics.com}

\section{Reviewed by Karen Bernd, Davidson College}

Submitted June 30, 2003; Revised July 11, 2003; Accepted July 11, 2003

Monitoring Editor: Sarah C.R. Elgin

What better way to clarify a topic and allow it to come alive than to provide students with a way to actually see it? Images, diagrams, and flowcharts can draw students in. Animated, interactive materials can provide an even bigger pull. Since my own skills in both drawing and interpretive dance are quite limited, I always look for multimedia containing strong background material and thought-provoking, timely topics. Materials that make the final cut must also meet other criteria: the presentation must be clear, I must be able to run the materials on my lecture hall computers and make them available for the students for viewing outside of class, and presentation and navigation ease are important. When considering use in undergraduate classes, the Roche Genetics: Education Program CD-ROM version 4.0 meets some, but not all, of these criteria. Its main shortcomings lie in its lack of proofreading.

\section{GENERAL ORGANIZATION}

The program is organized using a theme park analogy. An information booth leads to a central area connecting paths between five park areas. Topics include basic genetics, methods for studying monogenic and common complex disorders, pharmacogenetics, and a discussion of common questions concerning the ethics of research in such areas. I was pleasantly surprised to find that the focus is on education. Roche's official statement on genetics is provided and a Roche specific product is mentioned in one supporting method, but other than those two specific instances, it is not an F. Hoffman-La Roche infomercial. The material includes pharmacogenetics and there is a leaning toward the disease treatment and drug discovery focus of F. Hoffman-La Roche Ltd. This is fair since I would hope that authors write about their area of expertise. The medical angle is also a good way to catch students' interest. Many of the disorders mentioned are common ones that allow students to see the application of genetic theory and research in their lives.

Like a theme park, the five areas can be visited in any order. More than a layman's understanding of genetics is helpful when approaching pharmacogenetics, and so a progressive approach is implied but not required. The latter areas

DOI: $10.1187 /$ cbe.03-03-0011

Corresponding reviewer. E-mail address: kabernd@davidson. edu.

Note. This CD-ROM is available free of charge from HoffmanLa Roche and can be ordered from their web site at www. rochegenetics.com. provide highlighted links to earlier sections if viewers run across unfamiliar concepts. Because different, independent examples are used in each area, excerpts can be used in the classroom without having holes in the story. Other strengths in the program's organization include links on every page to a searchable dictionary and to a contents page containing a "Powerpoint-esque" index of all pages in the program. When I asked a recent graduate (Victoria Statler, '03) and a biology major (Ashley Wilson, '04) to provide student impressions of the program, they both mentioned the contents feature as a plus that allowed them to scan and choose topics. Other assets are the Did You Know? and Deeper Level information on many pages. Clicking Did You Know? provides interesting side facts about a topic such as why Northern blots are called Northern blots, the Greek derivation of Leptin, and the fact that the red vizcacha is the only nondiploid mammalian species (it is tetraploid). These tidbits are interesting asides and satisfy the trivia buff in me. Deeper Level information is used to provide details about topics such as the Human Genome Project, association studies, and molecular techniques and would be good as background material for lectures or labs. Areas can be toured without using this feature or the Did You Know? factoids, but using them adds to both the depth and the breadth of information.

\section{SECTION OVERVIEWS}

The Journey Through the Cell is the subtitle for the "Introduction to Genetics" section. Its inclusion shows that the authors realize that some viewers may need basic genetics background information. Covering all of introductory genetics in 77 pages and approximately 50 deeper-level topics is ambitious. Topics include defining what genes are, methods for determining genes and their functions, basic Mendelian inheritance, and recombination. I was pleased that attention was given to beneficial mutations and variations from Mendelian inheritance such as mitochondrial inheritance, genomic imprinting, and incomplete penetrance.

While I agree with the list of topics chosen, both my student reviewers and I were surprised at where emphases were placed. For example, the nucleotide structures are never shown. Since a significant number of pages discuss gene organization, DNA replication, PCR, and Northern blots, having more information on how all that hybridization occurs would be appropriate. I recognize, however, that Roche Genetics may have been either following a current trend in many 
biology textbooks that omits chemical structures or targeting an upper-level audience where such knowledge is assumed. The fact that the program quickly moves into advanced topics like microarrays and pharmacogenomics may suggest that the latter is the case. Another indication of an upper-level target audience is overall word choice. Pages include little text and extensive narration. The narration and the dictionary use terms that are likely to be confusing to the average introductory biology student. The script employs a dense, clause-laden sentence structure that would be hard to follow if this were your first exposure to the topic.

Users should be aware of mistakes, ranging from minor to major, that are found throughout the CD-ROM. A majority of them are found in this section. Minor errors include typographical ones, such as the use of "complimentary" when "complementary" is correct and a link that reads "AniPage10mal cloning." Also, some pages include Internet sites for additional information, and as luck would have it, a few of those sites are no longer active. Somewhat more serious are misleading diagrams, such as the replication figure with two leading strands (no Okazaki fragments!) (Fig. 1) and the PCR flowchart suggesting target fragments being amplified after one round of synthesis rather than three. My category of major errors includes those that would confuse students or things that just should not be done. Three such errors are found in this section; an interactive page on pedigrees where assigning a correct genotype results in feedback that you were incorrect, an animation of meiosis where DNA synthesis appears to occur in meiosis II, and a page on Mendelian inheritance where the first sentence is plagiarized from the provided reference link.

With this introduction to the basics, the second area delves into The Genetic Jungle: Finding Genes Associated with Disease. Using the identification of CFTR $\triangle F 508$ (cystic fibrosis; CF) and leptin (obesity) as examples, the program does a very good job of outlining pedigrees, positional cloning, and the use of animal models. While paths to discovery are presented as quite linear, the authors do infuse a sense of the amount of effort that was required.

The strongest parts are the clear flowcharts, discussion of genome scanning, and the way the presentation shows application of approaches defined in the first area. Again, errors are present. While the narration is correct, pedigrees showing inheritance of $C F$ and a chromosome 1 marker are shown with a LOD $>3$, incorrectly indicating linkage, and bad highlighting that shows a frameshift mutation instead of a deleted codon at F508 in CFTR. Also, the text says that $>800$ CFTR mutations are known and the narration reports 600 . This is unfortunate because the rest of the section is good. However, these types of errors would confuse introductory-level students, and their presence made the content of topics with which I was not as familiar become suspect. Fortunately, the next three areas seem to have had better proofreaders.

The Gene Pool: Genetics of Common Complex Disorders begins with a strong discussion of the interplay between genetics and environmental factors and why conditions can have multifactorial causes. Schizophrenia, Alzheimer's, lactose intolerance, atherosclerosis, and HIV are discussed so the information will work well with a variety of courses and approaches. I also appreciate the use of examples where the contribution

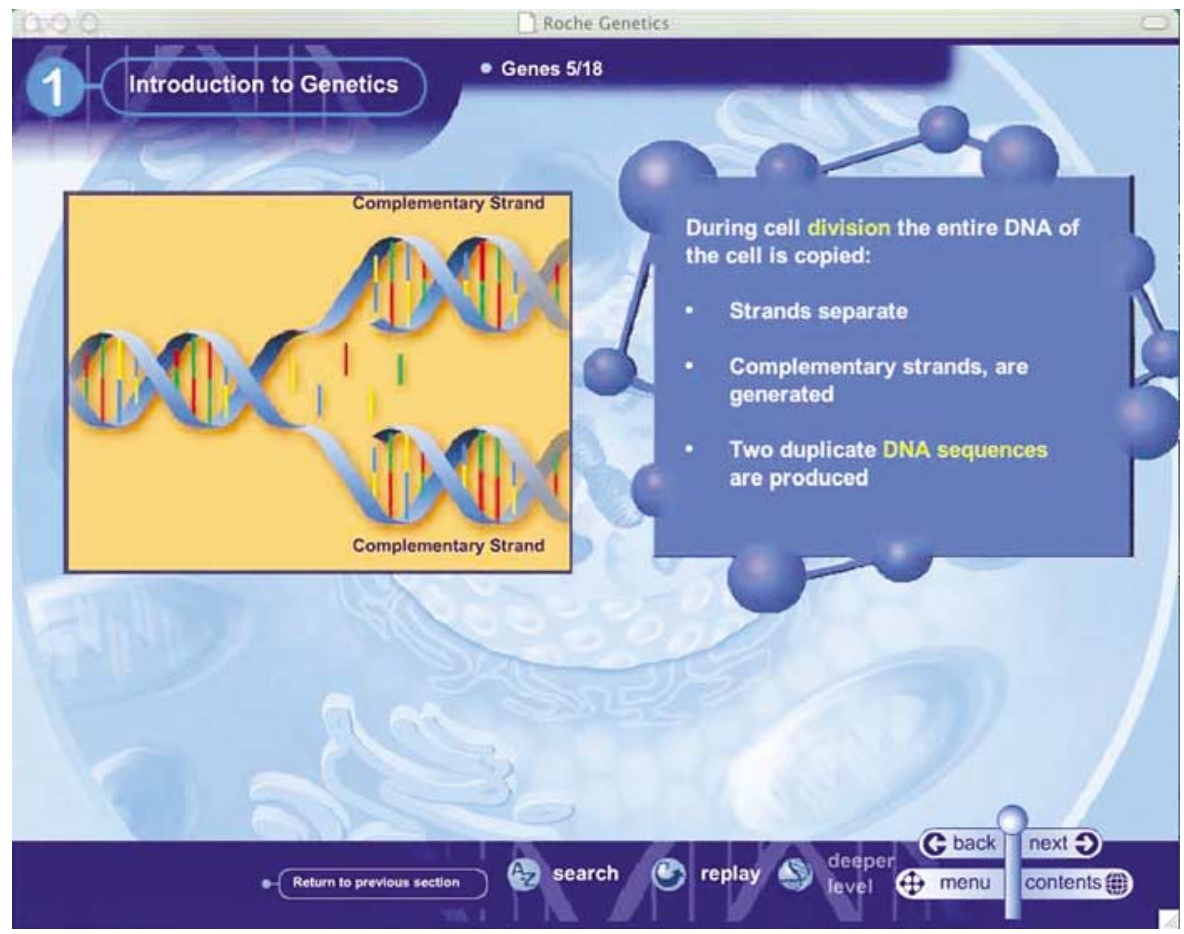

Figure 1. Diagram of DNA replication in which the process has been oversimplified so that it no longer contains a lagging strand. 


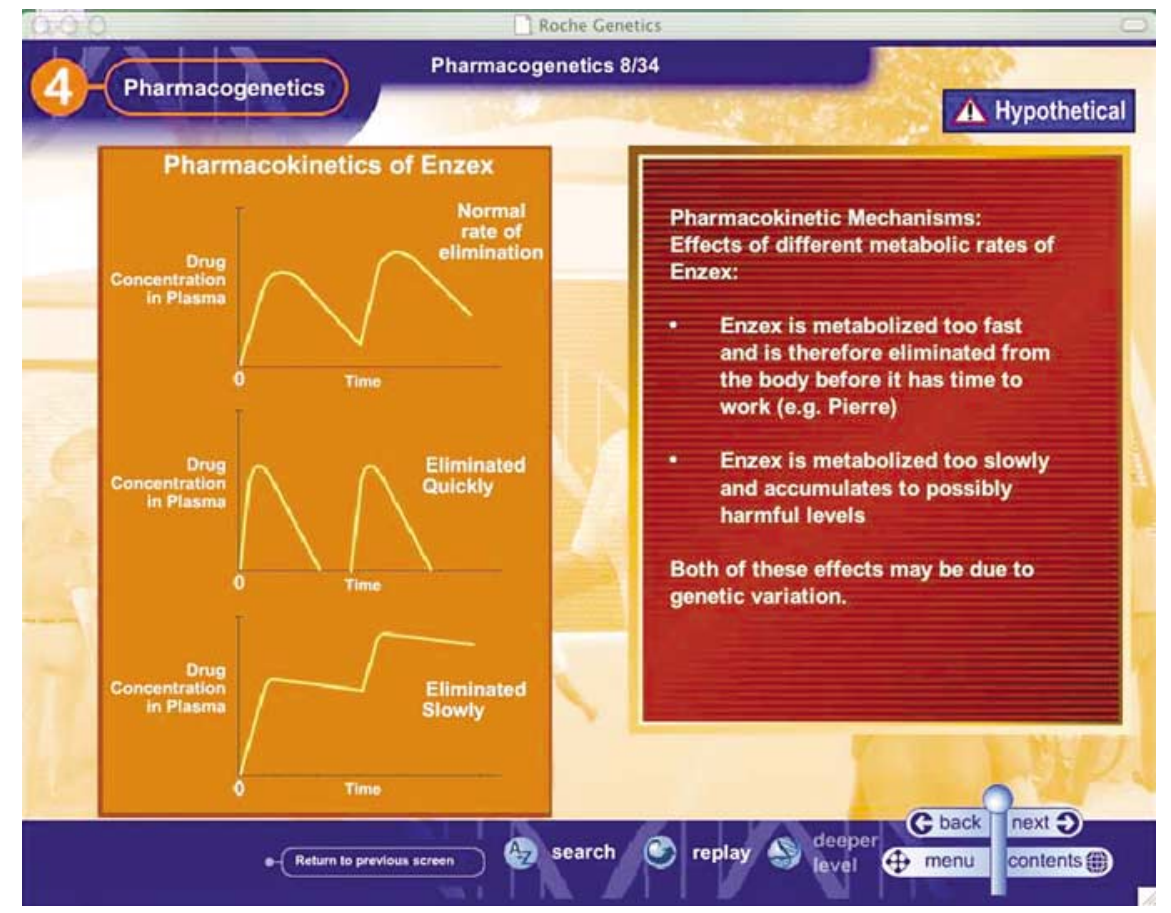

Figure 2. A page from the Pharmacogenetics section outlining how an individual's metabolism can change the effectiveness of a hypothetical antiasthma medication.

of lifestyle and environment can add new wrinkles and begin discussions.

I found The Gene Scene: Pharmacogenetics area interesting and refreshing: interesting because it is timely and effective, using a hypothetical blood pressure drug to explain this new type of research. Roche defines terms well and provides good supporting examples (Fig. 2). I found it refreshing because it varied from the slide show approach in two distinct ways. The area is a conversation between two men and an information kiosk whose female attendant does not have the traditional answering machine voice. We are never introduced but I am certain that she hails from northern New Jersey or Long Island. This woman narrates a vast majority of the section and the voice that departed from the expected made the section for me.

The final section (Ethical, Legal and Social Issues) also departs from the predicted. Perhaps heralding this departure, the section has no theme-park name and each page is topped with a large "6," in a theme park with only five areas. This probably should not seem odd in an ethics section that consistently misspells "beneficence," but it does suggest that a lack of attention to details persists. Again, these typographical errors are unfortunate because they detract from an otherwise strong and important section. While many are concerned about the interface between scientific research and ethics, few devote time or space to the discussion. As a closet bioethicist, I found a few of the definitions oversimplified, but the overall approach and scope quite good. Because of the authors (scientists) and producers (pharmaceutical company), this section could be used to challenge students to provide arguments supporting or refuting the program's stance or concerning other types of research.
Finally, this section's format is inconsistent with the rest. All other sections are a combination of narration, text, and diagrams. This section is text only. All others contain interactive questions. This section has none. As one student reviewer pointed out, in a CD-ROM based on a theme park you might expect a bit more interaction.

Because many lecture halls and computer labs do not allow users to install programs, I was very pleased to see that the program can be run from the disc if Flash 6 is on the computer. (Flash is on the disc if needed.) I own and teach using computers with various operating systems, ranging from OS9.0, 9.2, and OSX to Windows 98 and 2000 professional. The program ran on all computers except my home PC (Windows 98). I think this was because of my default AOL browser. On wired campuses this should not pose a problem. Because many of the diagrams are good, it is a bonus that all diagrams can be downloaded from the site listed in the "author and copyrights" section. The Roche Web site also includes other program facts; the program is free and is available in French, German, Spanish, English, and Chinese (Mandarin). Having all of these translations may explain (but not excuse) the sometimes dense wording, odd word choices, and misspellings.

Overall Version 4.0 has a strong underlying idea and good content, but it is in need of another round of content reviewing and proofreading. Because a majority of the errors are typographical or unclear representations, this version could be very usable supplemental material for upper-level undergraduates or as a review for early graduate students. It just supports caveat emptor for freeware. Documentation refers to the CD-ROM as a "living document" that will be updated. I expect that the next version will be stronger and I will keep an eye out for 4.1. 